\title{
METRICS ASSOCIATED WITH EXTREMAL PLURISUBHARMONIC FUNCTIONS
}

\author{
MACIEJ KLIMEK
}

(Communicated by Eric Bedford)

\begin{abstract}
A natural metric is introduced on the family of all polynomially convex compact $L$-regular sets in $\mathbb{C}^{n}$, thus turning this family into a complete metric space. An application in complex dynamics is described.
\end{abstract}

\section{INTRODUCTION}

Let $\|p\|_{E}$ denote the supremum norm of a polynomial $p: \mathbb{C}^{n} \longrightarrow \mathbb{C}$ on a compact set $E \subset \mathbb{C}^{n}$. In approximation theory it is often important to estimate the change of $\|p\|_{E}$ as $E$ changes. Since explicit evaluation of the supremum norms of polynomials can be laborious even in simple situations (see e.g. [AK2]), one looks for general qualitative methods. One approach is to use the generalized Bernstein-Walsh inequality (1.1) related to the Siciak extremal function

$$
\Phi_{E}(z)=\sup _{p}\left\{|p(z)|^{1 / \operatorname{deg} p}\right\}
$$

where the supremum is taken over all non-constant complex polynomials $p$ on $\mathbb{C}^{n}$ such that $\|p\|_{E} \leq 1$ (see [SI1], [SI2]). If $p$ is a polynomial which is not identically zero on $E$, one gets

$$
\frac{\|p\|_{F}}{\|p\|_{E}} \leq\left\|\Phi_{E}\right\|_{F}^{\operatorname{deg} p}
$$

for any compact set $F$. Despite being rather general, this inequality offers very good estimates in many cases (see e.g. [ABE]). It is usually difficult to calculate explicitly the Siciak extremal function of a particular set, but our knowledge concerning the behaviour of this function is quite extensive (see e.g. [KL2]). Note that the above estimate becomes trivial for small sets $E$ (if $E$ is pluripolar, then $\Phi_{E}=\infty$ outside $E$ ), and does not seem to be suitable for studying smaller families of polynomials (e.g. polynomials of a particular degree or with real coefficients). In a sense, the right-hand side of (1.1) measures

Received by the editors January 31, 1994.

1991 Mathematics Subject Classification. Primary 32F05, 31C10.

Key words and phrases. Extremal plurisubharmonic functions, pluricomplex Green functions, complex dynamics. 
a distance between $E$ and $F$. The purpose of this note is to show that, indeed, it can be turned into a metric and to discuss some consequences of this fact.

\section{THE METRIC $\Gamma$}

Throughout the paper, if $f$ is a complex-valued function on a set $S$, then $\|f\|_{S}$ denotes the supremum of $|f|$ on $S$. Let $(X, d)$ be a metric space and let $\mathscr{K}(X)$ denote the set of all (non-empty) compact subsets of $X$. Let $\delta_{E}(x)$ denote the distance from $x \in X$ to $E \in \mathscr{K}(X)$. The classical Hausdorff metric $\chi$ on $\mathscr{K}(X)$ can be defined by

$$
\chi(E, F)=\max \left\{\left\|\delta_{E}\right\|_{F},\left\|\delta_{F}\right\|_{E}\right\}=\left\|\delta_{E}-\delta_{F}\right\|_{X}
$$

In the context of pluripotential theory, if one wants to specify how far a point $z \in \mathbb{C}^{n}$ is from a compact set $E \subset \mathbb{C}^{n}$ it seems natural to use the pluricomplex Green function $V_{E}$ of $E$ (with pole at infinity) instead of the distance function $\delta_{E}$. Recall that

$$
V_{E}(z)=\sup \{u(z): u \in \mathscr{L}, u \leq 0 \text { on } E\},
$$

where $\mathscr{L}$ denotes the family of all plurisubharmonic functions $u$ on $\mathbb{C}^{n}$ such that $\sup \left\{u(z)-\log ^{+}\|z\|: z \in \mathbb{C}^{n}\right\}<\infty$. (For background material and references see [KL2].) If the set $E$ is compact, then the pluricomplex Green function of $E$ is the natural logarithm of Siciak's extremal function of $E$, i.e., $V_{E}=\log \Phi_{E}$.

A set $E \in \mathscr{K}\left(\mathbb{C}^{n}\right)$ is said to be $L$-regular if $V_{E}$ is continuous. We define a pseudometric $\Gamma$ on the set of all compact $L$-regular subsets of $\mathbb{C}^{n}$ as follows. If $E, F$ are two such sets, we put

$$
\Gamma(E, F)=\max \left\{\left\|V_{E}\right\|_{F},\left\|V_{F}\right\|_{E}\right\}=\left\|V_{E}-V_{F}\right\|_{E \cup F}=\left\|V_{E}-V_{F}\right\|_{\mathbb{C}^{n}} .
$$

Let $\mathscr{R}$ denote the set of all $L$-regular polynomially convex compact subsets of $\mathbb{C}^{n}$. The restriction of $\Gamma$ to $\mathscr{R} \times \mathscr{R}$ is a metric.

The polynomial estimate (1.1), after symmetrization of its right-hand side, can now be restated in terms of $\Gamma$ as follows. If $p: \mathbb{C}^{n} \longrightarrow \mathbb{C}$ is a complex polynomial and $E, F \in \mathscr{R}$, then

$$
\|p\|_{E} \leq \exp (\operatorname{deg} p \Gamma(E, F))\|p\|_{F} .
$$

For example, since explicit formulas for $V_{[-1,1]^{n}}$ and $V_{B_{1} \cap \mathbb{R}^{n}}$ are known (see [SII] and [LUN], respectively), it is not difficult to check that

$$
\Gamma\left([-1,1]^{n}, P_{1}\right)=\Gamma\left(B_{1} \cap \mathbb{R}^{n}, B_{1}\right)=\log (1+\sqrt{2}),
$$

where $B_{1}$ and $P_{1}$ denote the closed unit ball and polydisc in $\mathbb{C}^{n}$, respectively. Therefore

and

$$
\|p\|_{P_{1}} \leq(1+\sqrt{2})^{\operatorname{deg} p}\|p\|_{[-1,1]^{n}}
$$

$$
\|p\|_{B_{1}} \leq(1+\sqrt{2})^{\operatorname{deg} p}\|p\|_{B_{1} \cap \mathbb{R}^{n}}
$$

(See also Theorem 2.1 in $[\mathrm{ABE}]$ and a note at the end of that article.)

It is natural to ask to what extent the functions $V_{E}$ and $\delta_{E}$ are related. For a compact set $E$, if $\delta_{E}$ is subharmonic in the complement of $E$, then $E$ is convex (see [AK1]). (If $n=1$, it is enough to suppose that $\delta_{E}$ is subharmonic near $E$; see [PAR] for details.) This imposes a restriction on any potential 
relationship between these functions. Nevertheless, some estimates of $V_{E}$ in terms of $\delta_{E}$ exist. In the course of studying Markov's inequalities one looks at sets $E$ for which there are positive constants $M, m, \sigma$ such that

$$
V_{E} \leq M\left(\delta_{E}\right)^{m} \text { in } E_{\sigma},
$$

where $E_{\sigma}$ denotes the $\sigma$-dilation of $E$, i.e., the set $\left\{\delta_{E} \leq \sigma\right\}$. Such constants $M, m, \sigma$ have been shown to exist for large classes of sets $E$ (see [SI3], [P-P], [PL1], [PL2]).

Several other (pseudo)metrics related to $\Gamma$ can be defined. For instance, (2.1) defines a pseudometric on the family of non-pluripolar bounded subsets of $\mathbb{C}^{n}$. If $V_{E}$ and $V_{F}$ in (2.1) are replaced by their upper semicontinuous regularization, we obtain another pseudometric. A local analogue of $\Gamma$ (as well as of its variations, which we have just described) can also be obtained. Let $\Omega$ be an open set in $\mathbb{C}^{n}$ and let $E \subset \Omega$. Following Siciak [SI2] one defines the relative extremal function $h_{E, \Omega}$ by the formula

$$
h_{E, \Omega}(z)=\sup \{v(z): v \in \mathscr{P} \mathscr{S} \mathscr{C}(\Omega), v \mid E \leq 0, v \leq 1\} .
$$

Suppose that $\Omega$ is hyperconvex, i.e., it is a bounded domain which admits a continuous plurisubharmonic function $\varrho: \Omega \longrightarrow(-\infty, 0)$ such that $\varrho(z) \rightarrow 0$ if $z \rightarrow \partial \Omega$. Let $\mathscr{R}_{\Omega}$ be the family of all compact subsets $E$ of $\Omega$ such that $h_{E, \Omega}$ is continuous. Define

$$
\Gamma_{\Omega}(E, F)=\max \left\{\left\|h_{E, \Omega}\right\|_{F},\left\|h_{F, \Omega}\right\|_{E}\right\}=\left\|h_{E, \Omega}-h_{F, \Omega}\right\|_{\Omega}, \quad E, F \in \mathscr{R}_{\Omega} .
$$

We can also modify this pseudometric just as we have done in the case of $\Gamma$.

Sometimes $\Gamma$ and $\Gamma_{\Omega}$ can be linked via estimates. Let $\Omega \subset \mathbb{C}^{n}$ be a hyperconvex domain and let $K \in \mathscr{R} \cap \mathscr{K}(\Omega)$. Define

$$
M=\inf \left\{V_{K}: \mathbb{C}^{n} \backslash \Omega\right\}, \quad N=\sup \left\{V_{K}: \partial \Omega\right\} .
$$

Define also

$$
B_{\Gamma}(K, \varrho)=\{E \in \mathscr{R}: \Gamma(K, E) \leq \varrho\}, \quad \varrho>0 .
$$

If $0<\varrho<M$, then $B_{\Gamma}(K, \varrho) \subset \mathscr{K}(\Omega)$ and we have the following inequalities:

$$
(M-\varrho) \Gamma_{\Omega}(E, F) \leq \Gamma(E, F) \leq(N+\varrho) \Gamma_{\Omega}(E, F)
$$

for all $E, F \in B_{\Gamma}(K, \varrho)$.

The relative extremal functions transform well under non-degenerate holomorphic mappings (see e.g. Propositions 4.5.13 and 4.5.14 in [KL2]). If $f$ is such a mapping between two hyperconvex domains $\Omega_{1}, \Omega_{2}$, then $E \mapsto f(E)$ satisfies the Lipschitz condition with the constant 1 (with respect to $\Gamma_{\Omega_{1}}, \Gamma_{\Omega_{2}}$ ); if $f$ is proper, then $E \mapsto f^{-1}(E)$ is an isometry. The function $V_{E}$ and - consequently - the pseudometric $\Gamma$, because of their global character, are preserved only by proper polynomial mappings satisfying an extra condition. This will be put to use in the last section.

\section{COMPLETENESS AND OTHER PROPERTIES OF $\Gamma$}

It is well known that if $X$ is a complete metric space, then so is $(\mathscr{K}(X), \chi)$. The metric space $(\mathscr{R}, \Gamma)$ enjoys the same property. 
Theorem 1. The pair $(\mathscr{R}, \Gamma)$ is a complete metric space.

Proof. Let $B_{\varrho}$ denote the closed ball in $\mathbb{C}^{n}$ with centre at the origin and radius $\varrho>0$. Recall that $V_{B_{\varrho}}(z)=\log ^{+}(\|z\| / \varrho)$. If $\left\{E_{j}\right\}$ is a Cauchy sequence in $\mathscr{R}$, then $\left\{V_{E_{j}}-V_{B_{1}}\right\}$ is a Cauchy sequence in the Banach space $L^{\infty}\left(\mathbb{C}^{n}\right)$ and hence is uniformly convergent. Consequently $\left\{V_{E_{j}}\right\}$ converges uniformly on $\mathbb{C}^{n}$ to a function $f \in \mathscr{C}\left(\mathbb{C}^{n}\right) \cap \mathscr{L}$. We claim that $E=f^{-1}(0)$ is non-empty. Indeed, since $\left\{E_{j}\right\}$ is a Cauchy sequence, there exists a natural number $k$ such that

$$
V_{E_{k}}-1 / 2<V_{E_{j}} \text { for all } j \geq k .
$$

Choose $R>0$ such that $E_{k} \subset B_{R}$. Then

$$
\left\{V_{E_{j}}<1 / 2\right\} \subset\left\{V_{E_{k}}<1\right\} \subset\left\{V_{B_{R}} \leq 1\right\}=B_{R e} \text { for all } j \geq k .
$$

Suppose that $E=\varnothing$. Then there exists $\sigma>0$ such that $f>\sigma$ in $B_{R e}$. Consequently, if $j$ is sufficiently large, then $V_{E_{j}}>\sigma$ in $B_{R e}$ which is impossible because $E_{j}$ is the zero-set of $V_{E_{j}}$. To finish the proof we have to show that $f=V_{E}$. Clearly $f \leq V_{E}$. To prove the opposite inequality we first show that $\{\{f<\epsilon\}\}_{\epsilon>0}$ is a base of the filter of all neighbourhoods of the set $E$ in $\mathbb{C}^{n}$. We know from (3.1) that $V_{E_{k}}-1 / 2 \leq f$ in $\mathbb{C}^{n}$. Since $E_{k} \in \mathscr{R}$, one can find a constant $M$ such that $f(z) \geq M+\log (\|z\|+1)$ for all $z \in \mathbb{C}^{n}$. Choose $r>0$ so that $E \subset B_{r}, \delta_{E} \geq 1$ on $\partial B_{r}$ and $M+\log (r+1)>1$. We have to show that for each $\epsilon \in(0,1)$ there exists $\delta \in(0,1]$ such that $\{f<\delta\} \subset\left\{\delta_{E}<\epsilon\right\}$. Suppose this is not so. Then there is $\epsilon \in(0,1)$ such that for each positive integer $j$ one can find a point $z_{j}$ such that $f\left(z_{j}\right)<j^{-1}$ and $\delta_{E}\left(z_{j}\right)>\epsilon$. Note that $z_{j} \in B_{r}$ because outside this ball $f(z) \geq M+\log (\|z\|+1)>1$. Since $B_{r}$ is compact, there is a subsequence $\left\{z_{j_{l}}\right\}$ of the sequence $\left\{z_{j}\right\}$ which is convergent to a point $z_{0} \in B_{r}$ as $l \rightarrow \infty$. Then $0 \leq f\left(z_{0}\right)=\lim _{l \rightarrow \infty} f\left(z_{j_{l}}\right) \leq \lim _{l \rightarrow \infty} 1 / j_{l}=0$, and hence $z_{0} \in E$. On the other hand $\lim _{l \rightarrow \infty} \delta_{E}\left(z_{j_{l}}\right)=\delta_{E}\left(z_{0}\right) \geq \epsilon$, which is impossible. Finally, take $u \in \mathscr{L} \cap \mathscr{C}\left(\mathbb{C}^{n}\right)$ such that $u<0$ on $E$. Then there exists $\delta>0$ such that $\{f<\delta\} \subset\{u<0\}$. Consequently, if $j$ is sufficiently large, then $E_{j} \subset\{u<0\}$ and thus $u \leq V_{E_{j}}$ for all such $j$. Therefore $u \leq f$, and hence $V_{E} \leq f$ because $u$ was arbitrarily chosen.

Directly from the proof of the theorem we obtain the following corollary.

Corollary 1. If $E \in \mathscr{R}$, then the family $\left\{\left\{V_{E}<\epsilon\right\}\right\}_{\epsilon>0}$ forms a base of the filter of all neighbourhoods of the set $E$ in $\mathbb{C}^{n}$.

Similarly to the Hausdorff metric, the metric $\Gamma$ has the following set-theoretic property.

Corollary 2. If $A_{1}, \ldots, A_{k}, B_{1}, \ldots, B_{k} \in \mathscr{R}$, then

$$
\Gamma\left(A_{1} \cup \ldots \cup A_{k}, B_{1} \cup \ldots \cup B_{k}\right) \leq \max _{1 \leq j \leq k}\left\{\Gamma\left(A_{j}, B_{j}\right)\right\} .
$$

Moreover, if $A, B, C \in \mathscr{R}$ and $A \subset B \subset C$, then $\Gamma(A, B) \leq \Gamma(A, C)$.

Proof. It suffices to show (3.2) for $k=2$; the general case follows by induction. We have $\left\|V_{A_{1} \cup A_{2}}\right\|_{B_{1} \cup B_{2}} \leq \max \left\{\left\|V_{A_{1}}\right\|_{B_{1}},\left\|V_{A_{2}}\right\|_{B_{2}}\right\}$ and the last expression is not greater than the right-hand side of (3.2). A similar estimate holds for $\left\|V_{B_{1} \cup B_{2}}\right\|_{A_{1} \cup A_{2}}$. The second conclusion of the corollary is obvious. 
Condition (2.2) and other similar inequalities can sometimes imply simple estimates between $\chi$ and $\Gamma$. For instance, such estimates can be formulated for dilations of sets (i.e. sets of the form $\left\{\delta_{E} \leq \varepsilon\right\}$, where $E$ is a set and $\varepsilon>0$ ).

Corollary 3. Let $E, F, G \in \mathscr{R}$ and let $\epsilon, \kappa, \lambda$ be positive numbers. Then

$$
V_{E_{\epsilon}} \leq \log \left(\frac{\delta_{E_{\epsilon}}}{\epsilon}+1\right)
$$

and consequently

$$
\Gamma\left(F_{\kappa}, G_{\lambda}\right) \leq \log \left(\frac{\chi\left(F_{\kappa}, G_{\lambda}\right)}{\min \{\kappa, \lambda\}}+1\right) .
$$

It is easy to notice that if $E_{j} \rightarrow E$ in $(\mathscr{R}, \Gamma)$ and $E_{j} \rightarrow F$ in $\left(\mathscr{K}\left(\mathbb{C}^{n}\right), \chi\right)$, then $F \subset E$. Indeed, if $a \in F$, then one can find a sequence of points $a_{j} \in E_{j}$ such that $a_{j} \rightarrow a$ as $j \rightarrow \infty$. So $0 \leq V_{E}\left(a_{j}\right) \leq \Gamma\left(E, E_{j}\right) \rightarrow 0$ as $j \rightarrow 0$. Hence $V_{E}(a)=0$ as required.

Sometimes the set $F$ may be considerably smaller than $E$. Let $F=\{z \in \mathbb{C}$ : $|z|=1\}$, let $E$ denote the closed unit disc, and let $E_{j}=\left\{e^{i t}: t \in\left[0,2 \pi-j^{-1}\right]\right\}$ for $j=1,2, \ldots$. Then the sequence $\left\{E_{j}\right\}$ converges to $F$ in $(\mathscr{K}(\mathbb{C}), \chi)$. It also converges in $(\mathscr{R}, \Gamma)$ but this time the limit is $E$. More generally, if $\left\{E_{j}\right\}$ is an increasing sequence in $\mathscr{R}$ such that $F=\bigcup_{j=1}^{\infty} E_{j}$ is compact, then this sequence converges to $F$ with respect to $\chi$ and to the polynomially convex hull of $F$ with respect to $\Gamma$.

Observe that there are sequences which are convergent with respect to the Hausdorff metric but not with respect to $\Gamma$. For instance $\chi\left(B_{R}, B_{r}\right)=R-r$ for $R>r \geq 0$ (where $B_{0}=\{0\}$ ) and $\Gamma\left(B_{R}, B_{r}\right)=\log (R / r)$ for $R>r>0$. So, if $E_{j}=B_{R e^{-j}}$ for $j=1,2, \ldots$, then $E_{j} \rightarrow\{0\} \notin \mathscr{R}$ with respect to $\chi$, but $\Gamma\left(E_{j}, E_{j+1}\right)=1$ for all $\mathrm{j}$.

Corollary 4. Let $R>0$ and let $\mathscr{R}\left(B_{R}\right)=\mathscr{R} \cap \mathscr{K}\left(B_{R}\right)$. The metric spaces $(\mathscr{R}, \Gamma)$ and $\left(\mathscr{R}\left(B_{R}\right), \Gamma\right)$ are not compact.

The above examples suggest the following question. Suppose that $E_{j} \rightarrow E$ in $(\mathscr{R}, \chi)$. Is it true that $E_{j} \rightarrow E$ in $(\mathscr{R}, \Gamma)$ ? First of all, it is easy to see that a weaker assertion is always true: $\Gamma\left(F_{j}, E\right) \rightarrow 0$ as $j \rightarrow \infty$, where $\epsilon_{j}=\chi\left(E_{j}, E\right)$ and $F_{j}$ is the polynomially convex hull of the $\epsilon_{j}$-dilation of $E_{j}$. Secondly, because of the Caratheodory Convergence Theorem, the answer to the above question is affirmative in the one-dimensional case for connected sets (see Proposition 1 below). In $\mathbb{C}^{n}$, the answer is not known, except for some simple sequences of sets. For instance, the statement is true if $E_{j}=\bar{D}_{j}, E=\bar{D}$, where $D_{j}, D$ are domains which (as in the Caratheodory convergence) have the property that every $z \in D$ has a neighbourhood which lies in all $D_{j}$ for $j$ large enough.

Proposition 1. Suppose that $E_{j}, E \in \mathscr{R}$ are connected subsets of the complex plane containing the origin. If the sequence $\left\{E_{j}\right\}$ converges to $E$ with respect to $\chi$, then it also converges to $E$ with respect to $\Gamma$.

Proof. Let $h(z)=1 / z, D_{j}=h\left(\mathbb{C} \backslash E_{j}\right)$ and $D=h(\mathbb{C} \backslash E)$. Then the sequence $\left\{D_{j}\right\}$ converges in the sense of Caratheodory to its kernel $D$. The domains $D_{j}, D$ are simply connected. For each $j$, let $f_{j}$ be a conformal mapping from 
the unit disc onto $D_{j}$, such that $f_{j}(0)=0$ and $f_{j}^{\prime}(0)>0$. By the Caratheodory Convergence Theorem (see [DUR]), the sequence $\left\{f_{j}\right\}$ converges uniformly on compact subsets of the unit disc to a conformal mapping $f$ from the unit disc onto $D$. Furthermore, the sequence $\left\{f_{j}^{-1}\right\}$ converges uniformly on compact subsets of $D$ to $f^{-1}$. Therefore $V_{E_{j}} \rightarrow V_{E}$ as $j \rightarrow \infty$ locally uniformly on $\mathbb{C} \backslash E$ and hence the required statement is a consequence of the following lemma.

Lemma 1. Let $E_{j}, E \in \mathscr{R}$ be such that if $U$ is an open set containing $E$, then $E_{j} \subset U$ for all but finitely many $j$. The following conditions are equivalent.

(a) $V_{E_{j}} \rightarrow V_{E}$ uniformly in $\mathbb{C}^{n}$.

(b) $V_{E_{j}} \rightarrow V_{E}$ locally uniformly in the complement of $E$.

(c) $\lim \sup _{j \rightarrow \infty} V_{E_{j}}=0$ at each point of $E$.

Proof. To show (b) $\Longrightarrow$ (c) take $\varepsilon>0$. The set $U=\left\{V_{E}<\varepsilon\right\}$ is a bounded neighbourhood of $E$. There exists $j_{0}$ such that for all $j \geq j_{0}$ we have $V_{E_{j}} \leq 2 \varepsilon$ on $\left\{V_{E}=\varepsilon\right\}$. By the maximum principle the same estimate holds in $U$, and hence on $E$.

Suppose now that (c) is satisfied. Consider the functions $v=\lim \sup _{j \rightarrow \infty} V_{E_{j}}$ and $w=\sup _{j \geq 1} V_{E_{j}}$. Let ${ }^{*}$ denote the operation of taking the upper semicontinuous regularization of functions. Since $\{v=+\infty\}=\{w=+\infty\}$, it follows that $w^{*} \in \mathscr{L}$ (see e.g. [SI2] or [KL2]), and thus $v^{*} \in \mathscr{L}$. Hence the set $\left\{v<v^{*}\right\}$ is pluripolar [B-T], and consequently $v \leq V_{E}$ in $\mathbb{C}^{n}$. Now it suffices to apply the Hartogs lemma (in the open sets $\left\{V_{E}<\epsilon\right\}$ for $\epsilon>0$ ) to show that $\lim _{j \rightarrow \infty}\left\|V_{E_{j}}\right\|_{E}=0$. Since also $\lim _{j \rightarrow \infty}\left\|V_{E}\right\|_{E_{j}}=0$, we get (a).

Recall that the Robin constant $\gamma(E)$ of a set $E \subset \mathbb{C}^{n}$ is defined by the formula

$$
\gamma(E)=\limsup _{\|z\| \rightarrow \infty}\left(V_{E}^{*}(z)-\log \|z\|\right),
$$

where, as before, the asterisk denotes the upper semicontinuous regularization. The logarithmic capacity $c(E)$ of $E$ is then defined as $c(E)=\exp (-\gamma(E))$. We have the following property.

Corollary 5. If $E, F \in \mathscr{R}$, then $|\gamma(E)-\gamma(F)| \leq \Gamma(E, F)$. In particular the logarithmic capacity is continuous on $(\mathscr{R}, \Gamma)$.

\section{INVERSE ITERATION SYSTEMS}

A collection $\mathscr{P}=\left\{P_{1}, \ldots, P_{k}, S\right\}$ will be called an inverse iteration system if $S \in \mathscr{R} \cup \varnothing$ and each $P_{j}: \mathbb{C}^{n} \longrightarrow \mathbb{C}^{n}$ is a polynomial mapping such that for some $\delta>1$

$$
\liminf _{\|z\| \rightarrow \infty} \frac{\left\|P_{j}(z)\right\|}{\|z\|^{\delta}}>0, \quad j=1, \ldots, k .
$$

It will be shown below that such systems, together with the metric $\Gamma$, behave rather like hyperbolic iterated function systems with respect to the Hausdorff metric ([BAR]; see also [HUT] and [B-D]). If $\mathscr{P}$ is as above and $E \in \mathscr{R}$, then $\mathscr{P}(E)$ will denote the polynomially convex hull of the set

$$
S \cup \bigcup_{j=1}^{k} P_{j}^{-1}(E),
$$

and $\mathscr{P}^{j}$ will denote the $j$-th iteration of $\mathscr{P}: \mathscr{R} \longrightarrow \mathscr{R}$. 
Theorem 2. If $\mathscr{P}$ is an inverse iteration system, then there exists a unique set $J \in \mathscr{R}$ such that $\mathscr{P}(J)=J$. Furthermore, for any $E \in \mathscr{R}$ the sequence $\mathscr{P}^{j}(E)$ converges to $J$ in $(\Gamma, \mathscr{R})$.

Proof. Let $P \in\left\{P_{1}, \ldots, P_{k}\right\}$. By Proposition 4.8 in [KL1] (see also Theorem 5.3.1 in [KL2]) if $E \in \mathscr{R}$, then also $P^{-1}(E) \in \mathscr{R}$. Moreover

$$
\frac{1}{\operatorname{deg} P}\left(V_{E} \circ P\right) \leq V_{P^{-1}(E)} \leq \frac{1}{\delta}\left(V_{E} \circ P\right) .
$$

Hence the mapping $E \mapsto P^{-1}(E)$ is a contraction with respect to $\Gamma$ with the contraction ratio $1 / \delta$. Consequently, according to Corollary 2, the mapping that associates with each $E \in \mathscr{R}$ the set $\mathscr{P}(E)$ is a contraction of $\mathscr{R}$ with ratio $1 / \delta$. Therefore Theorem 1 combined with the Banach Contraction Principle implies the required statement.

The following corollary partly overlaps with a convergence result obtained in [H-P] (see Theorem 2.1, Example 1 in Section 6 and Proposition 6.1 in that paper).

Corollary 6. Let $P: \mathbb{C}^{n} \longrightarrow \mathbb{C}^{n}$ be a polynomial mapping satisfying (4.1) with some $\delta>1$. Let

$$
J_{P}=\left\{z \in \mathbb{C}^{n}:\left\{P^{j}(z)\right\}_{j \geq 1} \text { is bounded }\right\},
$$

where $P^{j}=P \circ \ldots \circ P$ (j times $)$. Then $J_{P} \in \mathscr{R}, P^{-1}\left(J_{P}\right)=J_{P}=P\left(J_{P}\right)$, and for any $E \in \mathscr{R}, \Gamma\left(\left(P^{j}\right)^{-1}(E), J_{P}\right) \rightarrow 0$ as $j \rightarrow \infty$.

Proof. In view of (4.2), $J_{P}=\mathbb{C}^{n} \backslash\left\{z \in \mathbb{C}^{n}: \lim _{j \rightarrow \infty}\left\|P^{j}(z)\right\|=\infty\right\}$, and there exists $R>0$ such that $J_{P}=\bigcap_{j \geq j_{0}}\left(P^{j}\right)^{-1}\left(B_{R}\right)$ for each positive integer $j_{0}$. Let $J$ be the fixed point of the contraction $\mathscr{P}=\{P, \varnothing\}$ (see Theorem 2). Then $P^{-1}\left(B_{R}\right)=\mathscr{P}\left(B_{R}\right)$ and $J \subset J_{P} \subset\left(P^{j}\right)^{-1}\left(B_{R}\right) \rightarrow J$ in $(\mathscr{R}, \Gamma)$ as $j \rightarrow \infty$.

Corollary 7. Let $P=\left(p_{1}, \ldots, p_{n}\right): \mathbb{C}^{n} \longrightarrow \mathbb{C}^{n}$ be a polynomial mapping satisfying (4.1) with $\delta=\operatorname{deg} p_{1}=\ldots=\operatorname{deg} p_{n}>1$. Then

$$
\inf \{\|\hat{P}(z)\|:\|z\|=1\} \leq c\left(J_{P}\right)^{1-\delta} \leq \sup \{\|\hat{P}(z)\|:\|z\|=1\},
$$

where $\hat{P}$ denotes the homogeneous part of $P$ of degree $\delta$ and $J_{P}$ is given by (4.3).

Proof. By (4.2), if $E \in \mathscr{R}$, then $M c\left(P^{-1}(E)\right)^{\delta} \leq c(E) \leq N c\left(P^{-1}(E)\right)^{\delta}$, where $M$ and $N$ denote the infimum and the supremum in (4.4) respectively. (Notice that

$$
\left.M=\liminf _{\|z\| \rightarrow \infty}\left(\|P(z)\| /\|z\|^{\delta}\right) \quad \text { and } \quad N=\limsup _{\|z\| \rightarrow \infty}\left(\|P(z)\| /\|z\|^{\delta}\right) .\right)
$$

Therefore $M c\left(\left(P^{j+1}\right)^{-1}(E)\right)^{\delta} \leq c\left(\left(P^{j}\right)^{-1}(E)\right) \leq N c\left(\left(P^{j+1}\right)^{-1}(E)\right)^{\delta}$. This implies the required estimates because of continuity of $c$ with respect to $\Gamma$.

If $n=1$, the last corollary reduces to Lemma 15.1 in [BRO].

\section{REFERENCES}

[ABE] R. Aron, B. Beauzamy, and P. Enflo, Polynomials in many variables. Real vs complex norms, J. Approx. Theory 74 (1993), 181-198.

[AK1] D. H. Armitage and Ü. Kuran, The convexity of a domain and the superharmonicity of the signed distance function, Proc. Amer. Math. Soc. 93 (1985), 598-600. 
[AK2] R. Aron and M. Klimek, Supremum norms for polynomials, preprint.

[B-D] M. Barnsley and S. Demko, Iterated function systems and the global construction of fractals, Proc. Roy. Soc. London Ser. A 399 (1985), 243-275.

[BAR] M. Barnsley, Fractals everywhere, Academic Press, Boston, 1988.

[B-T] E. Bedford and B. A. Taylor, A new capacity for plurisubharmonic functions, Acta Math. 149 (1982), 1-40.

[BRO] H. Brolin, Invariant sets under iteration of rational functions, Ark. Mat. 6 (1965), 103-144.

[DUR] P. L. Duren, Univalent functions, Springer-Verlag, New York, 1983.

[H-P] J. H. Hubbard and P. Papadopol, Superattractive fixed points in $\mathbb{C}^{n}$, Indiana Univ. Math. J. 43 (1994), 321-365.

[HUT] J. E. Hutchinson, Fractals and self similarity, Indiana Univ. Math. J. 30 (1981), 713-747.

[KL1] M. Klimek, Extremal plurisubharmonic functions and L-regular sets in $\mathbb{C}^{n}$, Proc. Roy. Irish Acad. Sect. A 82 (1982), 217-230.

[KL2] _ Pluripotential theory, Oxford Univ. Press, Oxford, 1991.

[LUN] M. Lundin, The extremal plurisubharmonic function for convex symmetric subsets of $\mathbb{R}^{n}$, Michigan Math. J. 32 (1985), 197-201.

[PAR] M. J. Parker, Convex sets and subharmonicity of the distance function, Proc. Amer. Math. Soc. 103 (1988), 503-506.

[P-P] W. Pawłucki and W. Pleśniak, Markov's inequality and $\mathscr{C} \infty$ functions on sets with polynomial cusps, Math. Ann. 275 (1986), 467-480.

[PL1] W. Pleśniak, Quasianalytic functions in the sense of Bernstein, Dissertationes Math. (Rozprawy Mat.) 147 (1977), 1-69.

[PL2] _ Markov's inequality and the existence of an extension operator for $\mathscr{C}^{\infty}$ functions, J. Approx. Theory 61 (1990), 106-117.

[SI1] J. Siciak, On some extremal functions and their applications in the theory of analytic functions of several complex variables, Trans. Amer. Math. Soc. 105 (1962), 322-357.

[SI2] _ Extremal plurisubharmonic functions in $\mathbb{C}^{n}$, Ann. Polon. Math. 29 (1981), 175-211.

[SI3] Highly noncontinuable functions on polynomially convex sets, Uni. Iagel. Acta Math. 25 (1985), 95-107.

Uppsala Universitet, Matematiska Institutionen, Box 480, S-751 06 Sweden

E-mail address: maciej@math.uu.se 